\title{
INFLUENCIA DEL ESFUERZO AXIAL EN COLUMNAS DE ESTRUCTURAS APORTICADAS DE HORMIGÓN ARMADO SUJETAS A ACCIONES SÍSMICAS SOBRE LA DERIVA DEL PISO
}

\author{
INFLUENCE OF AXIAL STRESS IN COLUMNS OF FRAME REINFORCED \\ CONCRETE STRUCTURES SUBJECT TO SEISMIC ACTIONS ON THE DRIFT OF \\ FLOORS
}

\section{ANTONIO LA TEGOLA', WALTER MERA²}

1 Universidad de Salento, Italia. antonio.lategola@unisalento.it

2 Universidad Católica de Santiago de Guayaquil. walter.mera@cu.ucsg.edu.ec

En algunas estructuras sometidas a acciones sísmicas los desplazamientos horizontales tienen una notable importancia. Los desplazamientos deben ser tomados en cuenta para evitar fenómenos de golpeteo con edificios contiguos y para asegurar la integridad de la superestructura. En las estructuras aporticadas de hormigón armado la intensidad de los esfuerzos axiales en las columnas adquieren mucha importancia, ya que para el comportamiento no lineal del material, el módulo elástico tangente se degrada notablemente. En las estructuras con columnas muy esbeltas, a pesar de que sean despreciables los efectos de segundo orden, la rigidez flexional, se reduce debido a que el módulo elástico tangente en las columnas, influenciado por el valor del esfuerzo axial, resulta menor al módulo elástico al origen. Los desplazamientos horizontales pueden por lo tanto no ser aceptables. En las estructuras con columnas esbeltas nacen los efectos de segundo orden que pueden conducir a un colapso. Algunas normativas recomiendan limitar las tensiones en las columnas de concreto con el fin de contener la influencia sobre los desplazamientos. En este trabajo, con referencia a las leyes constitutivas del concreto de tipo parábola/rectángulo, se examina la respuesta deformativa ya sea de columnas no esbeltas, 0 esbeltas bajo la acción de fuerzas transversales al aumentar la intensidad del esfuerzo axial. Algunos ejemplos numéricos evidencian este estudio. Además, siempre con referencia a algunos ejemplos, se muestra la influencia del esfuerzo axial sobre la deriva del piso de las estructuras aporticadas de hormigón armado.

PALABRAS CLAVE: pórticos, columnas esbeltas, deriva de pisos
ABSTRACT In some structures subjected to seismic actions horizontal displacements have a remarkable importance. The displacements must be taken into account to avoid knocking phenomena with adjacent buildings and to ensure the integrity of the superstructure. In reinforced concrete structures, the intensity of the axial forces in the columns becomes very important, since for the non-linear behavior of the material, the elastic modulus tangent degrades considerably. In the structures with very slender columns, although the effects of second order are negligible, the flexional rigidity is reduced because the elastic modulus tangent in the columns, influenced by the value of the axial effort, is less than the elastic modulus to the origin. Horizontal displacements may therefore not be acceptable. In the structures with slender columns the second order effects are born that can lead to a collapse. Some regulations recommend limiting tensions in concrete columns in order to contain the influence on the displacements. In this work, with reference to the constitutive laws of the parabola / rectangle type concrete, the deformative response of either non-slender or slender columns under the action of transverse forces is examined by increasing the intensity of the axial stress. Some numerical examples show this study. In addition, always with reference to some examples, the influence of axial stress on the drift of the floor of reinforced concrete structures is shown.

KEYWORDS: pórticos, columnas esbeltas, deriva de pisos 


\section{INTRODUCCIÓN HIPOTESIS}

En el diseño de las estructuras de hormigón armado sismorresistentes, dos estados límites asumen una importancia notable. Un estado límite último con el fin de garantizar la seguridad respecto a la supervivencia, y un estado límite de trabajo para evitar daños a la superestructura.

En las estructuras aporticadas la seguridad de los miembros individuales depende obviamente de la geometría de las secciones y de la cantidad de armadura metálica. Para lo cual un dimensionamiento adecuado de las secciones y de la armadura metálica garantizan la resistencia y por tanto la supervivencia en ocasión del sismo de diseño.

En cuanto concierne la condición de trabajo, la misma está ligada a los desplazamientos que la estructura puede alcanzar bajo las acciones sísmicas; son importantes los desplazamientos horizontales ya sea de entre plano o absolutos, para evitar la rotura de paredes de mampostería y eventuales fenómenos de golpeteo con edificios contiguos. La deformación está sustancialmente ligada a la calidad del concreto y al estado de solicitaciones.

El problema está estrechamente conectado a la rigidez de los elementos verticales, columnas, pilares. Siendo el concreto un material de comportamiento inelástico, el módulo elástico se degrada al aumentar las solicitaciones. Para este fin las varias normativas indican algunas limitaciones del esfuerzo de compresión respecto a la sección del concreto, que en la condición de trabajo siente en medida reducida la contribución de la armadura metálica.

Por tanto es conveniente poner en evidencia cuanto sea efectivamente relevante la intensidad del esfuerzo axial agente respecto a la deformación bajo acciones transversales.

MÓDULO TANGENTE

Con referencia a una ley constitutiva del concreto de tipo parábola/rectángulo (figura 1), en el tramo parabólico, la ley está dada por

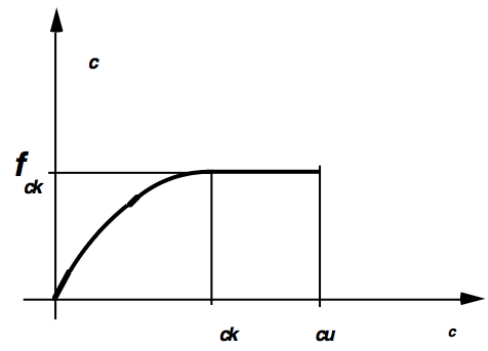

Figura 1. Ley constitutiva del concreto.

$$
\sigma_{c}=\frac{2 f_{c k}}{\varepsilon_{c k}}\left(\varepsilon_{c}-\frac{\varepsilon_{c}^{2}}{2 \varepsilon_{c k}}\right)
$$

El módulo tangente resulta:

$$
E_{t}=\frac{\partial \sigma_{c}}{\partial \varepsilon_{c}}=\frac{2 f_{c k}}{\varepsilon_{c k}}\left(1-\frac{\varepsilon_{c}}{\varepsilon_{c k}}\right)
$$

y el módulo al origen para $\varepsilon_{\mathrm{c}}=0$ está dado por

$$
E_{0}=\frac{2 f_{c k}}{\varepsilon_{c k}}
$$

Invirtiendo la (1) se tiene:

$$
\varepsilon_{c}=\varepsilon_{c k}\left(1-\sqrt{1-\frac{\sigma_{c}}{f_{c k}}}\right)
$$

y utilizando la (2) y la (3) se puede escribir:

$$
E_{t}=E_{0} \sqrt{1-\frac{\sigma_{c}}{f_{c k}}}
$$

En el caso de una sección de área A, solicitada a compresión, se puede escribir también

$$
E_{t}=E_{0} \sqrt{1-\frac{N_{c}}{N_{c k}}}
$$

habiendo puesto

$$
N_{c k}=A f_{c k}
$$

Debe observarse que para $\sigma_{c}=0,4 f_{c k}$ se tiene

$$
E_{t}=2 \frac{f_{c k}}{\varepsilon_{c k}} 0,776=1,549 \frac{f_{c k}}{\varepsilon_{c k}} \quad \frac{E_{t}}{E_{0}}=0,889
$$

Para el módulo elástico secante se obtiene:

$$
\begin{aligned}
\varepsilon_{c} & =\varepsilon c k(1-\sqrt{1-0,4})=0,225 \varepsilon c k \\
E_{s} & =\frac{0,4}{0,225} \frac{f_{c k}}{\varepsilon_{c k}}=1,778 \frac{f_{c k}}{\varepsilon_{c k}} \quad \frac{E_{s}}{E_{0}}=0,889 \\
\frac{E_{s}}{E_{0}} & =\frac{0,776}{0,889}=0,882
\end{aligned}
$$

La (6) para varios valores de la relación $\frac{N_{c}}{N_{c k}}$ proporciona $\frac{E_{t}}{E_{0}}$ de la cual se puede notar que para $\frac{N_{c}}{N_{c k}}$ se tiene un degrado del módulo $\frac{E_{t}}{E_{0}}=0,632$

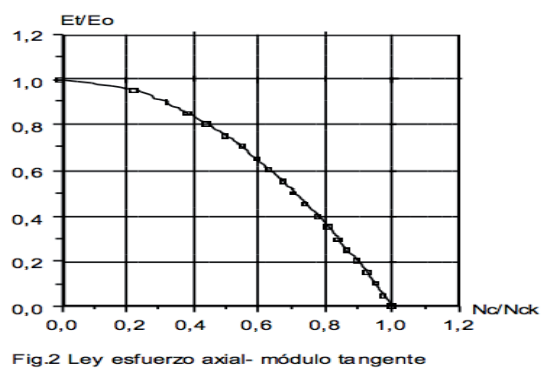

Figura 2. Ley esfuerzo axial - módulo tangente. 
TABLA 1.

\begin{tabular}{rrrr}
\hline NC/NCK & ET/EO & NC/NCK & ET/EO \\
\hline 0,00 & 1,000 & 0,55 & 0,671 \\
0,05 & 0,975 & 0,60 & 0,632 \\
0,10 & 0,949 & 0,65 & 0,592 \\
0,15 & 0,922 & 0,70 & 0,548 \\
\hline 0,20 & 0,894 & 0,75 & 0,500 \\
0,25 & 0,866 & 0,80 & 0,447 \\
0,30 & 0,837 & 0,85 & 0,387 \\
0,35 & 0,806 & 0,90 & 0,316 \\
0,40 & 0,775 & 0,95 & 0,224 \\
0,45 & 0,742 & 1,00 & 0,000 \\
0,50 & 0,707 & & \\
\hline
\end{tabular}

Por tanto, debido a la rigidez flexional de las columnas, solicitadas por un esfuerzo normal, se degrada la misma cantidad, resulta evidente que los desplazamientos horizontales son mayores de aquellos validados no teniendo en cuenta el valor del módulo tangente.

DESPLAZAMIENTOS HORIZONTALES EN COLUMNAS NO ESBELTAS

Con referencia al esquema de la figura 3 , la deformación en la parte superior, haciendo despreciables los efectos del segundo orden, son dados por la expresión:

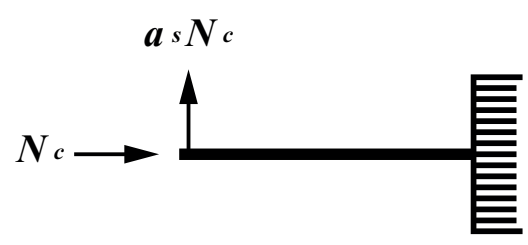

Figura 3. Columna empotrada en la base bajo acciones sísmicas horizontales.

$$
\delta=a_{s} N_{c} \frac{l^{3}}{3 E_{t} l}
$$

siendo as un coeficiente que tiene en cuenta la aceleración sísmica horizontal. La (8) puede también escribirse: y poniendo

$$
\frac{E_{t}}{E_{0}}=\sqrt{1-\frac{N_{c}}{N_{c k}}} \quad E_{0}=\frac{2 f_{c k}}{\varepsilon_{c k}}
$$

se obtiene la relación

$$
\delta \frac{1}{a_{s}} \frac{E_{0}}{N_{c k}} \frac{3 l}{l^{3}}=\frac{\frac{N_{c}}{N_{c k}}}{\sqrt{1-\frac{N_{c}}{N_{c k}}}}
$$

de la cual se obtiene la cantidad:

$$
\delta=\lambda^{2} a_{s} l \frac{\varepsilon_{c k} \frac{N_{c}}{N_{c k}}}{6 \sqrt{1-\frac{N_{c}}{N_{c k}}}}
$$

habiendo puesto $\lambda=\frac{l}{\rho}$

con $\rho$ radio de inercia de la sección en el plano de inflexión. Para evidenciar la no linealidad se puede

analizar la cantidad adimensional independiente de las características geométricas

$$
\bar{\delta}=\frac{\delta}{a_{s} l} \frac{l}{\lambda^{2}}=\frac{\varepsilon_{c k} \frac{N_{c}}{N_{c k}}}{6 \sqrt{1-\frac{N_{c}}{N_{c k}}}}
$$

De la tabla y del gráfico de la Figura 4 asumiendo:

$$
\varepsilon_{c k}=0,002 \text { concreto de clase } \leq C 50 / 60
$$

se puede notar la fuerte no linealidad; para $\frac{N_{c}}{N_{c k}}=0,6$ desplazamientos son 1,54 mayores de aquellos validados con el módulo al origen.

\section{En el gráfico y en la tabla se tiene:}

$$
d=\bar{\delta} x 1000
$$

\section{TABLA 2.}

\begin{tabular}{rrrr} 
NC/NCK & D & NC/NCK & D \\
\hline 0,00 & 0.0000 & 0,50 & 0.2357 \\
0,05 & 0.0171 & 0,55 & 0.2733 \\
0,10 & 0.0351 & 0,60 & 0.3162 \\
0,15 & 0.0542 & 0,65 & 0.3662 \\
\hline 0,20 & 0.0745 & 0,70 & 0.4260 \\
0,25 & 0.0962 & 0,75 & 0.5000 \\
0,30 & 0.1195 & 0,80 & 0.5963 \\
0,35 & 0.1447 & 0,85 & 0.7316 \\
0,40 & 0.1721 & 0,90 & 0.9487 \\
0,45 & 0.2023 & 0,95 & 14.162 \\
\hline
\end{tabular}

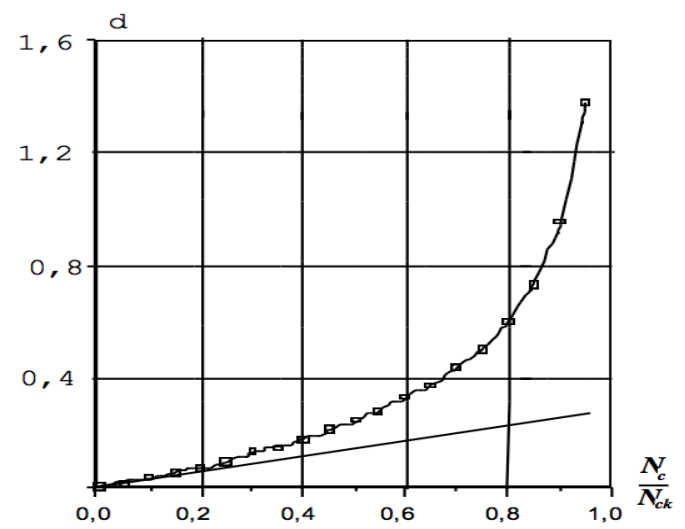

Figura 4. Ley esfurzoaxial-desplazamentoenlapartesuperior. 
DESPLAZAMIENTOS HORIZONTALES EN COLUMNAS SEGÚN LOS EUROCÓDIGOS

Según los Eurocódigos se puede adoptar el módulo elástico de cálculo secante validado por

$\sigma_{c}=0,4 f_{c k}$

poniendo

$E_{c d}=E_{s}$

se puede escribir: $\frac{\delta}{a_{s} l} \frac{1}{\lambda^{2}}=\frac{f_{c k}}{3 E_{c d}} \frac{N_{c}}{N_{c k}}$

se tiene un comportamiento de tipo lineal.

Para el cual a igualdad del valor de $a_{s} \frac{N_{c}}{N_{c k}}$,

en el caso del módulo tangente los desplazamientos son influenciados por el esfuerzo axial.

La cantidad

$$
\frac{\varepsilon_{c k}}{6 \sqrt{1-\frac{N_{c}}{N_{c k}}}}
$$

hace intervenir la

cualidad del concreto a través de la deformación $\varepsilon_{c k}$ y el valor del esfuerzo axial.

Según los Eurocódigos la cantidad $\frac{f_{c k}}{3 E_{c d}}$

depende solo de la calidad del concreto. Además los Eurocódigos proporcionan una limitación al valor del esfuerzo axial. Se considera no obstante que para la validación de los módulos de deba hacer referencia a la tensión media última del concreto, proporcionada por

$$
\begin{aligned}
& f_{c k}=0,83 R_{c k} \\
& f_{c m}=f_{c k}+8(\mathrm{~N} / \mathrm{mm} 2)
\end{aligned}
$$

DESPLAZAMIENTOS HORIZONTALES EN COLUMNAS

ESBELTAS

En el caso de columnas esbeltas se hace referencia al esquema de la figura 5:

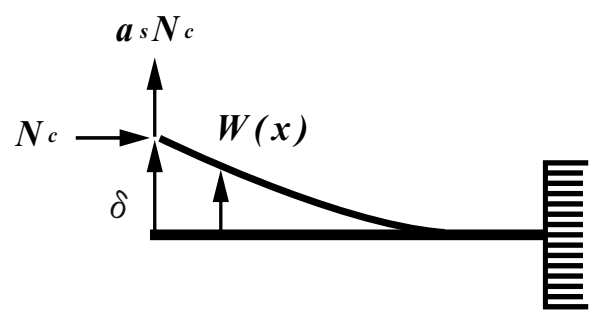

Figura 5. Esquema columna esbelta.

la ecuación de la deformada se escribe

$$
E_{t} I w^{\prime \prime}=\left[N_{c}(\delta-w(x))+(l-x) k N_{c}\right]
$$

y por doble derivación se convierte en:

$$
E_{t} I w^{I V}(x)+N_{c} w^{\prime \prime}(x)=0
$$

La integral general resulta

$$
w(x)=A+B x+C \sin \alpha x+D \cos \alpha x
$$

habiendo puesto

$$
\alpha^{2}=\frac{N_{c}}{E_{t} I}
$$

Las condiciones de borde son:

$$
\left\{\begin{array}{l}
w(0)=0 \\
w^{\prime}(0)=0 \\
w^{\prime \prime}(0)=0 \\
E_{t} I w^{\prime \prime \prime}(l)=-N_{c}\left(a_{s}+w^{\prime}(l)\right)
\end{array}\right.
$$

Las primeras dos proporcionan

$$
A=-D
$$

$B=-\alpha C$

Se puede escribir por tanto

$$
w(x)=A(1-\cos \alpha x)+B\left(x-\frac{1}{\alpha} \sin \alpha x\right)(15)
$$$$
w^{\prime \prime}(l)=\alpha(A \alpha \cos a \alpha l+B \sin \alpha l)=0
$$

de la cual se deriva $A=-\frac{B}{\alpha} \operatorname{tg} \alpha l$

y por tanto

$w(x)=-\frac{B}{\alpha}(\operatorname{tg} \alpha l(1-\cos \alpha x)-\alpha x+\sin \alpha x)$

resulta

$$
\begin{aligned}
& w^{\prime}(l)=B(-t g \alpha l \sin \alpha l+1-\cos \alpha l) \\
& w^{\prime \prime}(l)=-B \alpha^{2}(-t g \alpha l \sin \alpha l-\cos \alpha l)
\end{aligned}
$$

La cuarta condición se puede escribir

$$
w^{\prime \prime \prime}(l)=-\alpha^{2}\left(a_{2}+w^{\prime}(l)\right)
$$

se tiene por tanto

$$
\begin{aligned}
& -B \alpha^{2}(-t g \alpha l \sin \alpha l-\cos \alpha l)= \\
& -\alpha^{2}\left(a_{s}+B(\operatorname{tg} \alpha l \sin \alpha l+1-\cos \alpha l)\right) \\
& B=a_{s}
\end{aligned}
$$

por tanto

$w(x)=a_{s} \frac{1}{\alpha}(\operatorname{tg} \alpha l(1-\cos \alpha x)-\alpha x+\sin \alpha x)(16)$

El desplazamiento en la parte superior está dado por

$$
\delta=a_{s} \frac{(\operatorname{tg} \alpha l(1-\cos \alpha l)-\alpha l+\sin \alpha l)}{\alpha}
$$

también puede escribir 


$$
\frac{\delta}{a_{s} l}=\frac{(\operatorname{tg} \alpha l(1-c i s \alpha l)-\alpha l+\sin \alpha l)}{\alpha l}
$$

De la (13) se obtine

$$
\begin{aligned}
& \alpha^{2} l^{2}=\frac{N_{c}}{E_{t} I} l^{2}=\frac{N_{c}}{E_{t} A \rho^{2}} l^{2}= \\
& =\lambda^{2} \frac{N_{c}}{A E_{0} \sqrt{1-\frac{N_{c}}{N_{c k}}}}=\lambda^{2} \frac{\frac{N_{c}}{N_{c k}}}{\frac{A}{N_{c k}} E_{0} \sqrt{1-\frac{N_{c}}{N_{c k}}}}
\end{aligned}
$$

siendo

$$
\frac{N_{c k}}{A}=f_{c k} \text { e } E_{0}=\frac{2 f_{c k}}{\varepsilon_{c k}}
$$

se considera por último

$$
\alpha l=\lambda \sqrt{\frac{\frac{\varepsilon_{c k}}{2} \frac{N_{c}}{N_{c k}}}{\sqrt{1-\frac{N_{c}}{N_{c k}}}}}
$$

Por tanto es posible obtener de la (18) los valores de $\frac{\delta}{a_{s} l}$ para valores de la relación $\lambda=\frac{l}{\rho}$ al variar de $\frac{N_{c}}{N_{c k}}$

A los fines de problemas de inestabilidad d e 1 equilibrio la esbeltez referida a la longitud libre de inflexión, para el caso examinado, vale

$$
\lambda_{o}=\frac{l_{o}}{\rho}=2 \lambda
$$

Para evidenciar los efectos del segundo orden se puede considerar la cantidad

$$
\begin{aligned}
& \bar{\delta}=\frac{\delta}{a_{s} l} \frac{1}{\lambda^{2}}= \\
& =\frac{1}{\lambda^{2}} \frac{(\operatorname{tg} \alpha l(1-\cos \alpha l)-\alpha l+\sin \alpha l)}{\alpha}
\end{aligned}
$$

y confrontarla con la relación (9).

Si no se desprecian los efectos del segundo orden se obtienen, para $\varepsilon_{c k}=0,002$ concreto de clase $\leq C 50 / 60$

los valores son reportados (tabla 3 ) siendo, en $d=\delta \times 1000$ la última columna se reportan los valores sin los efectos del segundo orden.

\section{TABLA 3 DESPLAZAMIENTOS PARA VARIOS VALORES DE NC/NCK}

\section{$d=\delta \times 1000$}

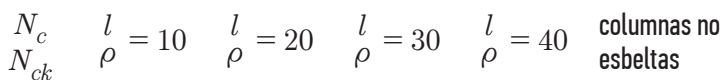

$\begin{array}{llllll}0.05 & 0.0171 & 0.0172 & 0.0174 & 0.0177 & 0.0171 \\ 0.10 & 0.0353 & 0.0357 & 0.0365 & 0.0377 & 0.0351 \\ 0.15 & 0.0546 & 0.0557 & 0.0576 & 0.0605 & 0.0542\end{array}$

\section{$d=\delta \times 1000$}

$\begin{array}{lllll}N_{c} & l \\ N_{c k} & \rho\end{array}=10 \quad \stackrel{l}{\rho}=20 \quad \stackrel{l}{\rho}=30 \quad \stackrel{l}{\rho}=40 \begin{aligned} & \begin{array}{l}\text { columnas no } \\ \text { esbeltas }\end{array} \\ & \end{aligned}$

\begin{tabular}{llllll}
0.25 & 0.0973 & 0.1009 & 0.1074 & 0.1181 & 0.0962 \\
0.30 & 0.1213 & 0.1268 & 0.1373 & 0.1553 & 0.1195 \\
0.35 & 0.1473 & 0.1555 & 0.1716 & 0.2006 & 0.1447 \\
0.40 & 0.1758 & 0.1877 & 0.2115 & 0.2576 & 0.1721 \\
0.45 & 0.2073 & 0.2240 & 0.2590 & 0.3317 & 0.2023 \\
0.50 & 0.2426 & 0.2658 & 0.3165 & 0.4326 & 0.2357 \\
0.55 & 0.2826 & 0.3146 & 0.3883 & 0.5793 & 0.2733 \\
0.60 & 0.3287 & 0.3729 & 0.4813 & 0.8147 & 0.3162 \\
\hline 0.65 & 0.3831 & 0.4445 & 0.6078 & 12.609 & 0.3662 \\
0.70 & 0.4490 & 0.5359 & 0.7929 & 24.582 & 0.4260 \\
\hline 0.75 & 0.5319 & 0.6585 & 10.958 & & 0.5000 \\
0.80 & 0.6423 & 0.8366 & 17.003 & & 0.5963 \\
0.85 & 0.8020 & 11.301 & 36.259 & & 0.7316 \\
0.90 & 10.707 & 17.502 & & & 0.9487 \\
\hline
\end{tabular}

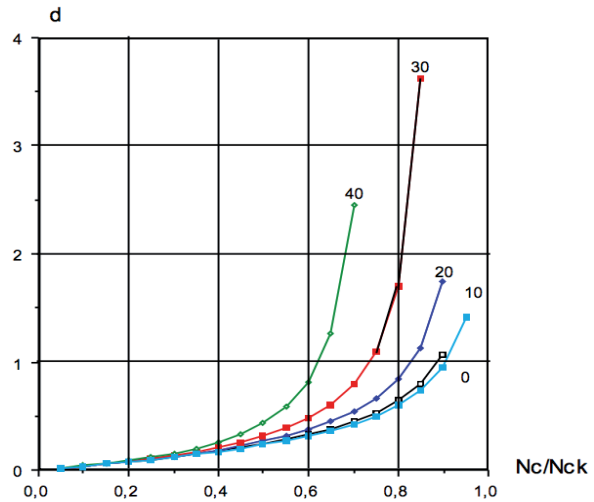

Figura 6. Comparación de los desplazamientos horizontales para varios valores de $\frac{l}{\rho}$.

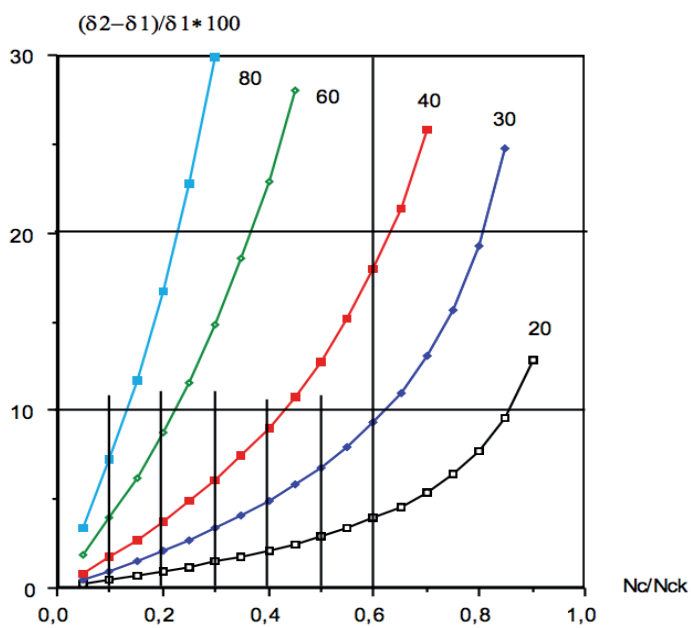

Figura 7. Porcentuales de los desplazamientos de segundo orden para varios valores de $\frac{l_{o}}{\rho}$ 
De la Tabla 3 y de los gráficos de la figura 6 se nota que los efectos del segundo orden comienzan a ser importantes para valores de $N_{c} / N_{c k}>0,5$

Indicando con $N_{c} / N_{c k}>0,5$ los valores de la esbeltez con respecto a la longitud libre de inflexión, en el gráfico de la figura 7 se reportan la diferencia porcentual entre los desplazamientos del segundo orden y aquellos del primer orden. Se puede notar que queriendo contener la diferencia entorno al $10 \%$, solo para la esbeltez $\lambda_{0}=\frac{l_{o}}{\rho}<30$ la relación $N_{c} / N_{c k}$ puede ser ob-

tenida alrededor de 0,6 ; en todos los otros casos es necesario contener dicha relación a valores inferiores para poder despreciar los desplazamientos del segundo orden.

Según los Eurocódigos, para valorar los desplazamientos teniendo en cuenta los efectos del segundo orden, en la (21) se debe poner:

$$
\alpha l=\lambda \sqrt{\frac{f_{c k} \frac{N_{c}}{N_{c k}}}{E_{c d}}}
$$

en el cual $N_{c}$ es el valor del esfuerzo axial aplicado y $a_{s} N_{c}$ la fuerza horizontal reportada al valor come alícuota del esfuerzo axial.

\section{EJEMPLOS NUMÉRICOS}

Se considera una columna de sección $40 \times 40 \mathrm{~cm}$ y de altura $400 \mathrm{~cm}$ con un concreto con $f_{c k}=25 \frac{\mathrm{N}}{\mathrm{mm}^{2}}$ solicitada por

$$
N_{c}=2000 K N \quad a_{s} N_{c}=100 K N
$$

$$
\begin{aligned}
& \text { Resulta } a_{s}=\frac{100}{2000}=0,05 \\
& \frac{N_{c}}{N_{c k}}=\frac{2000000}{25 x 400 x 400}=0,5 \\
& \frac{l}{\rho}=\frac{400}{40} \sqrt{12=34,64}
\end{aligned}
$$

despreciando los efectos de segundo orden, se tiene:

$$
\begin{aligned}
& \delta=\lambda^{2} a_{s} l \frac{\varepsilon_{c k} \frac{N_{c}}{N_{c k}}}{6 \sqrt{1-\frac{N_{c}}{N_{c k}}}}= \\
& 34,64^{2} \times 0,05 \times 400 \times 0,002 \\
& \times \frac{0,5}{6 \sqrt{1-0,5}}=5,66
\end{aligned}
$$

Considerando los efectos de segundo orden, se tiene:

$$
\begin{aligned}
\alpha l & =\lambda \sqrt{\frac{\frac{\varepsilon_{c k}}{2} \frac{N_{c}}{N_{c k}}}{\sqrt{1-\frac{N_{c}}{N_{c k}}}}}= \\
& =34 m 64 \sqrt{\frac{0,002 / 2 \times 0,5}{\sqrt{1-0,5}}}=0,921 \\
\delta & =a_{s} l \frac{(\operatorname{tg} \alpha l(1-\cos \alpha l)-\alpha l+\sin \alpha l)}{\alpha l}=8,58
\end{aligned}
$$

Según las indicaciones de los Eurocódigos, se obtiene, despreciando los efectos de segundo orden,

$$
\begin{aligned}
& f_{c m}=f_{c k}+8=33 \frac{\mathrm{N}}{\mathrm{mm}^{2}} \\
& E_{c m}=22000\left(\frac{f_{c m}}{10}\right)^{0,3}=31476 \frac{\mathrm{N}}{\mathrm{mm}^{2}} \\
& E_{c d}=1,2 \times 31476=37771 \frac{\mathrm{N}}{\mathrm{mm}^{2}} \\
& \delta=a_{s} l \lambda^{2} \frac{f_{c k}}{3 E_{c d}} \frac{N_{c}}{N_{c k}}=2,65 \mathrm{~cm}
\end{aligned}
$$

Siendo el esfuerzo axial de larga duración, es más correcto poner $E_{c m}$ en lugar de $E_{c d^{*}}$.

En dicho caso se tiene:

$$
\delta=a_{s} l \lambda^{2} \frac{f_{c k}}{E_{c m}} \frac{N_{c}}{N_{c k}}=3,18 \mathrm{~cm}
$$

Considerando los efectos de segundo orden resulta,

$$
\begin{aligned}
& \alpha l=\lambda \sqrt{\frac{f_{c k} \frac{N_{c}}{N_{c k}}}{E_{c d}}}=34,64 \sqrt{\frac{25 \times 0,5}{37771}}=0,630 \\
& \delta=a_{s} l \frac{(\operatorname{tg} \alpha l(1-\cos \alpha l)-\alpha l+\sin \alpha l)}{\alpha l}=3,15 \mathrm{~cm}
\end{aligned}
$$

Utilizando en su lugar $E_{c m}$ se tiene $\alpha l=0,690$

$\delta=3,93 \mathrm{~cm}$

Del ejemplo examinado resulta evidente la necesidad de tener en cuenta el módulo tangente.

Los desplazamientos así valoradas son $\mathrm{mu}^{-}$ cho mejores ya sea con los efectos del segundo orden, ya sea sin los efectos del segundo orden.

\section{CÁLCULO DE LOS DESPLAZAMIENTOS HORIZONTALES EN LOS PISOS TENIENDO EN CUENTA LA INFLUENCIA DE LOS ESFUERZOS AXIALES EN LAS COLUMNAS}

Para el cálculo de las deformaciones en algunos estructuras aporticadas, despreciando los efectos de segundo orden, se puede utilizar el módulo secante para las vigas en el cual el esfuerzo 
axial es generalmente muy pequeño; en las columnas en cambio es necesario tener en cuenta la influencia del esfuerzo axial y considerar el módulo tangente, que normalmente resulta inferior al módulo secante. En dicho caso, debido a que las rigideces flexionales vienen reducidas del módulo tangente, se obtienen desplazamientos horizontales más grandes. Cuando es necesario tener en cuentas los efectos de segundo orden el cálculo puede ser realizado con métodos no lineales tipo pushover. Con algunos ejemplos numéricos se demostrará la diferencia entre los desplazamientos obtenidos teniendo en cuenta o no la influencia del esfuerzo axial.

Se considera el pórtico plano reportado a continuación (figura 8). Para todas las vigas se asume el módulo secante, para las columnas se asume el módulo tangente es menor que el módulo secante.
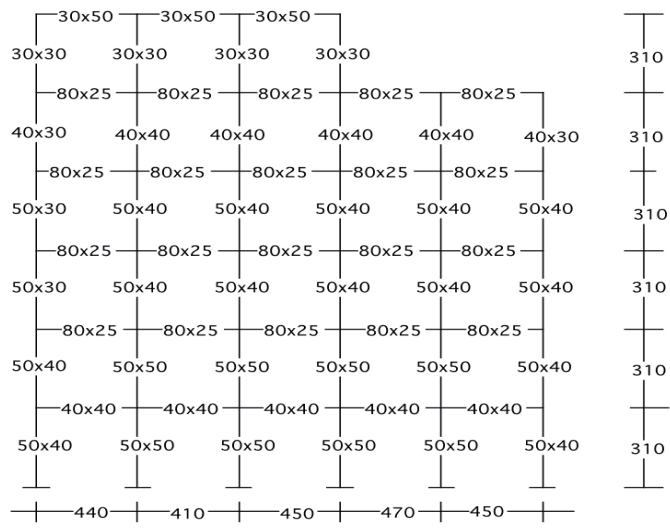

Figura 8. Geometría de la estructura (Medidas en $\mathrm{cm}$.).

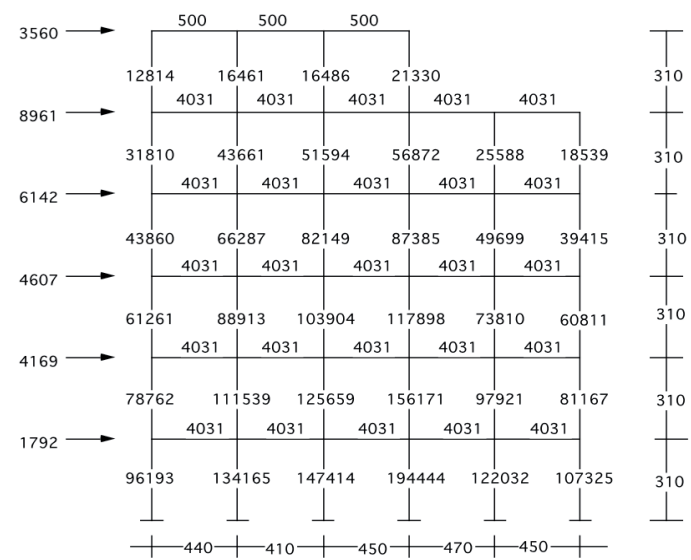

Figura 9. Esfuerzos axiales en las columnas y acciones en las vigas (Esfuerzon en daN, acciones en la vigas en daN/m).

$$
\begin{aligned}
& f_{c k}=0,83 R_{c k} \quad f_{c m}=f_{c k}+8\left(\mathrm{~N} / \mathrm{mm}^{2}\right) \\
& \text { para } R_{c k}=30 \frac{\mathrm{N}}{\mathrm{mm}^{2}}
\end{aligned}
$$

resulta

$$
\begin{aligned}
& E_{s}=\frac{0,4}{0,225} \frac{f_{c m}}{\varepsilon_{c k}}=1,778 \frac{f c m}{\varepsilon c k} \text { se tiene } \\
& E_{s}=29244 \frac{\mathrm{N}}{\mathrm{mm}^{2}}
\end{aligned}
$$

Considerando para toda la estructura el módulo secante, para la columnas se puede escribir $\frac{E_{t}}{E_{s}}=\frac{E_{o}}{E_{s}} \sqrt{1-\frac{N}{N_{k}}}$ y siendo $\frac{E_{o}}{E_{s}}=2 \frac{0,225}{0,4}=1,125$

se puede introducir una inercia ficticia igual a $I_{f}=I\left(1,125 \sqrt{1-\frac{N}{N_{k}}}\right) \leq I$

La valoración de los desplazamientos bajo el sismo de diseño viene calculados a partir de aquellos obtenidos del análisis lineal según las siguientes expresiones:

$$
\begin{aligned}
& d_{E}= \pm \mu d \cdot d_{E e} \\
& \text { con } \\
& \mu d=q \text { se } T_{l}<T_{c} \\
& \mu_{d}=1+(q-1)-T_{c} / T_{l} \quad \text { se } T_{l}<T_{c} \\
& \mu_{d} \leq 5 q-4
\end{aligned}
$$

siendo

Tl periodo propio de la estructora

$q$ factor de estructura

Tc el periodo correspondiente al inicio del tramo con velocidad constante del espectro.

La estructura examinada es caracterizada por los siguientes parámetros:

$$
T_{l}=0,50 \sec T_{C}=0,66 \sec q=3,9
$$

siendo $T_{l}<T_{C}$ se tiene

$$
\mu_{d}=1+(3,9-1) \cdot \frac{0,66}{0,50}=4,828
$$

Los desplazamientos entre pisos $d_{r} \leq 0,01 h$ no proporcionan daños a la infraestructura. Sin tener en cuenta la influencia del esfuerzo axial se obtienen los siguientes valores:

Piso 1 Desplazamiento relativo $0.23 \mathrm{~cm}$ Desplazamiento absoluto $0.23 \mathrm{~cm}$

Piso 2 Desplazamiento relativo $0.46 \mathrm{~cm}$ Desplazamiento absoluto $0.69 \mathrm{~cm}$

Piso 3 Desplazamiento relativo $0.61 \mathrm{~cm}$ Desplazamiento absoluto $1.30 \mathrm{~cm}$

Piso 4 Desplazamiento relativo $0.60 \mathrm{~cm}$ Desplazamiento absoluto $1.90 \mathrm{~cm}$

Piso 5 Desplazamiento relativo $0.38 \mathrm{~cm}$ Desplazamiento absoluto $2.28 \mathrm{~cm}$

Piso 6 Desplazamiento relativo $0.28 \mathrm{~cm}$ Desplazamiento absoluto $2.56 \mathrm{~cm}$ 
Los desplazamientos bajo la acción del sismo resultan, siendo

$$
\begin{aligned}
& \mu_{d}=1+(q-1)-T_{C} / T_{l} \quad \text { se } T_{l}<T_{C} \\
& T_{l}=0,50 \mathrm{sec} \\
& T_{c}=0,66 \mathrm{sec} \\
& q=3,9 \\
& \mu_{d}=1+(3,9-1) \cdot \frac{0,66}{0,50}=4,828
\end{aligned}
$$

Piso 1 Desplazamiento relativo $1.11 \mathrm{~cm}$ Desplazamiento absoluto $1.11 \mathrm{~cm}$ Piso 2 Desplazamiento relativo $2.22 \mathrm{~cm}$ Desplazamiento absoluto $3.33 \mathrm{~cm}$ Piso 3 Desplazamiento relativo $2.95 \mathrm{~cm}$ Desplazamiento absoluto $6.27 \mathrm{~cm}$ Piso 4 Desplazamiento relativo $2.90 \mathrm{~cm}$ Desplazamiento absoluto $9.17 \mathrm{~cm}$ Piso 5 Desplazamiento relativo $1.83 \mathrm{~cm}$ Desplazamiento absoluto $11.00 \mathrm{~cm}$ Piso 6 Desplazamiento relativo $1.35 \mathrm{~cm}$ Desplazamiento absoluto $12.07 \mathrm{~cm}$

El desplazamiento de deriva máximo permitido es $d_{r} \leq 0,01 * 310=3,10 \mathrm{~cm}$ y por tanto los valores así valorados resultan dentro del límite. Teniendo en cuenta la influencia del esfuerzo axial se obtienen los siguientes valores:

\begin{tabular}{lll} 
Piso 1 & Desplazamiento relativo $0.24 \mathrm{~cm}$ & Desplazamiento absoluto $0.24 \mathrm{~cm}$ \\
\hline Piso 2 & Desplazamiento relativo $0.47 \mathrm{~cm}$ & Desplazamiento absoluto $0.71 \mathrm{~cm}$ \\
\hline Piso 3 & Desplazamiento relativo $0.61 \mathrm{~cm}$ & Desplazamiento absoluto $1.32 \mathrm{~cm}$ \\
\hline Piso 4 & Desplazamiento relativo $0.60 \mathrm{~cm}$ & Desplazamiento absoluto $1.92 \mathrm{~cm}$ \\
\hline Piso 5 & Desplazamiento relativo $0.39 \mathrm{~cm}$ & Desplazamiento absoluto $2.31 \mathrm{~cm}$ \\
\hline Piso 6 & Desplazamiento relativo $0.30 \mathrm{~cm}$ & Desplazamiento absoluto $2.61 \mathrm{~cm}$
\end{tabular}

Los desplazamientos bajo la acción del sismo resultan:

\begin{tabular}{lll} 
Piso 1 & Desplazamiento relativo $1.16 \mathrm{~cm}$ & Desplazamiento absoluto $1.16 \mathrm{~cm}$ \\
\hline Piso 2 & Desplazamiento relativo $2,27 \mathrm{~cm}$ & Desplazamiento absoluto $3.43 \mathrm{~cm}$ \\
\hline Piso 3 & Desplazamiento relativo $2.95 \mathrm{~cm}$ & Desplazamiento absoluto $6.38 \mathrm{~cm}$ \\
\hline Piso 4 & Desplazamiento relativo $2.90 \mathrm{~cm}$ & Desplazamiento absoluto $9.28 \mathrm{~cm}$ \\
\hline Piso 5 & Desplazamiento relativo $1.88 \mathrm{~cm}$ & Desplazamiento absoluto $11.16 \mathrm{~cm}$ \\
\hline Piso 6 & Desplazamiento relativo $1.45 \mathrm{~cm}$ & Desplazamiento absoluto $12.61 \mathrm{~cm}$
\end{tabular}

La estructura en examen ha sido diseñada para mantener el valor del esfuerzo axial dentro de los límites indicados por las normativas y por tanto los desplazamientos de deriva que resultan están dentro de los límites permitidos.
CONCLUSIONES

El problema de una correcta valoración de los desplazamientos horizontales reviste una notable importancia con el fin de limitar los daños a la superestructura o de fenómenos de golpeteo con edificios contiguos en condiciones sísmicas. Por tanto en las estructuras aporticadas se debe tener en cuenta el estado de esfuerzos en las columnas por efecto de la solicitación axial, solicitación que en el momento que ocurre el sismo está presente como de larga duración. Para poder despreciar los desplazamientos de segundo orden, es además necesario limitar la esbeltez a más del valor del esfuerzo axial.

Además en función de los esfuerzos axiales en alguna columna se puede introducir el valor del módulo tangente correspondiente que resulta menor de aquel considerado para las vigas. Operado de esta manera es posible valorar los desplazamientos horizontales que seguramente son más reales y por tanto se puede obtener una mayor garantía respecto de la seguridad.

\section{REFERENCIAS BIBLIOGRÁFICAS}

1. Antonio La Tegola, Walter Mera "Diseño de las Estructuras de Hormigón Armado a los Estados Límites" UCSG (2013).

2. Commission of the European Communities (1991) "Eurocode N.2 Desingn of Concrete Structures".

3. Ministero delle Infrastrutture e dei Trasporti (Roma) (2008) "Norme Tecniche per le Costruzioni". 